\title{
Expression of $\mathrm{Cx} 43$ and Pax 3 proteins in the human placental villi and decidua during early pregnancy
}

\author{
Jinping Zhang ${ }^{\mathrm{a},{ }^{*}}$, Leijia Zhang ${ }^{\mathrm{a}}$, Xuehong Liu ${ }^{\mathrm{a}}$ and Fuqun Zhou ${ }^{\mathrm{b}}$ \\ ${ }^{a}$ Department of Histology and Embryology, Medical College, Shaoxing University, Shaoxing 312000, \\ Zhejiang, China \\ ${ }^{\mathrm{b}}$ Department of Obstetrics and Gynecology, Shaoxing Municipal Maternal and Children Hospital, \\ Shaoxing 312000, Zhejiang, China
}

\begin{abstract}
To study the expression and roles of Connexin43 (Cx43) and Paired-box3 (Pax3) proteins in the human Chorionic villi and decidua during early pregnancy, $\mathrm{Cx} 43$ and Pax3 protein expression in the human placental villi and decidua at 5-7 weeks of early pregnancy was studied by immunohistochemistry (SABC). Integrated optical density (IOD) of Cx43 and Pax3 protein expression was obtained by using special image analysis software. Cx43 and Pax3 proteins were expressed in all trophoblastic cells, cells of villi central axis and decidual cells. IOD of $\mathrm{Cx} 43$ and Pax3 proteins expressed in the syncytiotrophoblast decreased from 5 to 7 weeks, while Cx43 and Pax3 expressed in cytotrophoblast cells and decidual cells showed increased IOD during the same period. The differences between any two groups were statistically significant $(\mathrm{P}<0.05) . \mathrm{Cx} 43$ and $\mathrm{Pax} 3$ proteins were expressed during differentiation and development of placental villous cells in early phases of pregnancy. We propose that $\mathrm{Cx} 43$ and Pax3 may participate in endometrial decidualization and the regulation of trophoblastic invasion and differentiation. It is likely that $\mathrm{Cx} 43$ and Pax3 play critical roles in cell proliferation and differentiation during the processes of chorionic villi initiation/development and placental morphogenesis.
\end{abstract}

Keywords: Connexin43, pax3, Chorionic villi, decidua, Immunohistochemistry

\section{Introduction}

The placenta is an important organ that connects the developing fetus to the mother for material exchange. It provides suitable intrauterine environment for fetal growth. The normal development and function of the placenta is the key factor affecting the normal development of the fetus. Syncytiotrophoblast cells are critical cells in the placenta, secreting human chorionic gonadotropin (HCG), human chorionic somatomammotropin (HCS), progesterone and estrogen. During pregnancy, the syncytiotrophoblast fuse with the underlying cytotrophoblast cells and it contributes to the main physiological function of the placenta. $\mathrm{Cx} 43$ and Pax3 are regulatory proteins having close relationship with early development and formation of the placenta [1]. There are only few studies on

\footnotetext{
${ }^{*}$ Corresponding author: Jinping Zhang, Department of Histology and Embryology, Medical College, Shaoxing University, Shaoxing 312000, Zhejiang, China. Tel.: 0575-88345821; Fax: 0575-88062799; E-mail: pjzxuan@126.con.
}

0959-2989/14/\$27.50 @ 2014 - IOS Press and the authors. 
the protein expression of $\mathrm{Cx} 43$ and Pax 3 in the human placental villi and decidua during early pregnancy. In the present study, we investigated the expression of $\mathrm{Cx} 43$ and Pax3 in the human placental villi and decidua during early pregnancy by the SABC method of immunohistochemistry and further studied their roles in chorial generation/development and placental morphogenesis.

\section{Materials and methods}

\subsection{Subjects and groupsw}

Decidual and chorionic samples were collected from 26 adult women, who were diagnosed pregnant and requested induced abortion in Shaoxing municipal maternal and children hospital from January to February, 2007. All these women were between 5 and 7 weeks of pregnancy. Among them, 8, 10 and 8 cases were at 5, 6 and 7 weeks of pregnancy, respectively. The age ranged from 20 to 30 years old. They had regular menstrual cycle and knew accurate date for the last menstrual period. Moreover, all these patients did not have a history of abdominal pain and vaginal bleeding. They never took any hormone medicine.

\subsection{Methods}

\subsubsection{Main reagents}

Anti-Cx43 and anti-Pax3 polyclonal antibodies and the accessory kits were all purchased from Wuhan Boster Biological Technology Co., Ltd.

\subsubsection{Immunohistochemical staining}

Fresh chorionic villi and decidual tissues were fixed for 24 hours in $10 \%$ formalin. Samples were then rinsed with distilled water for $30 \mathrm{~min}$. Next, tissue blocks were routinely dehydrated, embedded in paraffin and cut to $4 \mu \mathrm{m}$ thickness slides. Four slides were prepared for each sample. The immunohistochemical SABC method was used for staining. The main steps were as follows: deparaffinization and rehydration $\rightarrow$ inactivation of endogenous enzymes with $3 \%$ hydrogen peroxide $\rightarrow$ heat-induced epitope retrieval $\rightarrow$ blocking with normal goat serum without wash $\rightarrow$ add the primary antibodies $\rightarrow$ biotinylation of goat serum $\rightarrow$ ABC (the slides were washed $3 \times 5$ min with PBS after each step) $\rightarrow$ add DAB to stain slides $\rightarrow$ hematoxylin staining. Next, the slides were dehydrated, transparentized, mounted and observed using a light microscopy.

\subsubsection{Controls}

PBS was used to replace the primary antibodies as the negative control. Available positive slides were used as the positive control.

\subsubsection{Data analysis}

The stained $\mathrm{Cx} 43$ and Pax 3 proteins were yellow or brown granules. $\mathrm{Cx} 43$ protein was dominantly located in the cytoplasm and cell membrane, while Pax 3 was distributed in the cytoplasm, nucleus and cell membrane. Five fields were randomly selected from each slide. The true color image analysis system 4.0 (Image processing center of Beihang University) was applied to determine the integral optical density (IOD) values of $\mathrm{Cx} 43$ and Pax3 protein expression in a certain visual sight. The average IOD of 5 visual sights for each section was taken as the sample IOD. 


\subsubsection{Statistical analysis}

The SPSS 12.0 software was used for statistical analysis. The calculated data were presented as "mean \pm SD". One-Way ANOVA was applied to compare data between each group. $\mathrm{P}<0.05$ was considered as statistically significant differences.

\section{Results}

\subsection{Cx43 expression in the human placental villi and decidua during early pregnancy}

Cx43 was mainly distributed in the cytoplasm and cell membrane of placental trophoblast cells and decidual cells. From 5 to 7 weeks of pregnancy, the IOD of Cx43 protein expressed in the syncytiotrophoblast decreased gradually (Figures 1 and 2), and the differences between 5 and 6 weeks were statistically significant $(\mathrm{P}<0.05)$. However, $\mathrm{Cx} 43$ in cytotrophoblast cells (Figures 1 and 2$)$ and decidual cells (Figures 3 and 4) showed increased IOD from 5 to 7 weeks, and the differences were statistically significant $(\mathrm{P}<0.05)$ (Table 1$)$.

\section{Table 1}

Cx43 expression in the human placental villi and decidua during early pregnancy

\begin{tabular}{lllll}
\hline \multirow{2}{*}{$\begin{array}{l}\text { Group number of } \\
\text { cases }\end{array}$} & Length of pregnancy & \multicolumn{4}{l}{ The average of positive Pax3 IOD (mean \pm SD) } \\
\cline { 3 - 5 } & & Syncytiotrophoblast & Cytotrophoblast & Decidual cells \\
\hline 8 & 5 weeks & $2936.79 \pm 287.56$ & $2331.57 \pm 291.20$ & $2291.11 \pm 292.89$ \\
\hline 10 & 6 weeks & $2497.50 \pm 320.65$ & $2627.30 \pm 362.23$ & $2699.35 \pm 394.73$ \\
\hline 8 & 7 weeks & -- & $2954.78 \pm 360.71$ & $2941.70 \pm 494.29$ \\
\hline $\mathrm{F}$ & 9.123 & 6.660 & 5.375 \\
\hline $\mathrm{P}$ & 0.008 & 0.005 & 0.012 \\
\hline
\end{tabular}

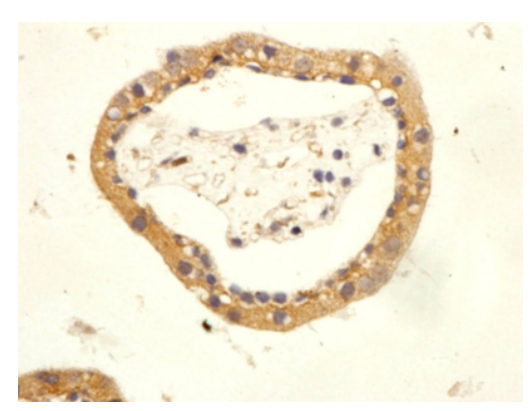

Fig. 1. Cx43 protein expression in the human placental trophoblast at 5 weeks of pregnancy, $\mathrm{SABC} \times 400$.

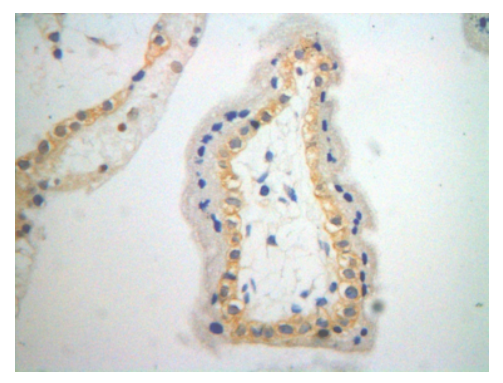

Fig. 2. Cx43 protein expression in the human placental villus trophocyte at 7 weeks of pregnancy, $\mathrm{SABC} \times 400$. 


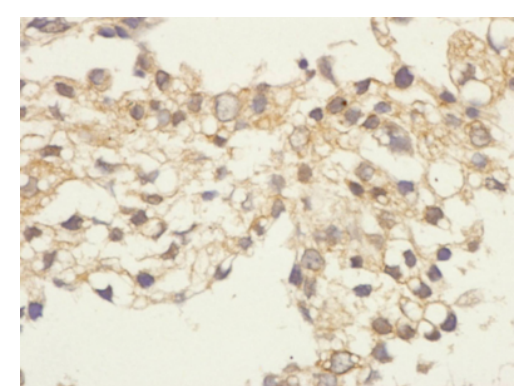

Fig. 3. $\mathrm{Cx} 43$ protein expression in the human decidual cells at 5 weeks of pregnancy, $\mathrm{SABC} \times 400$.

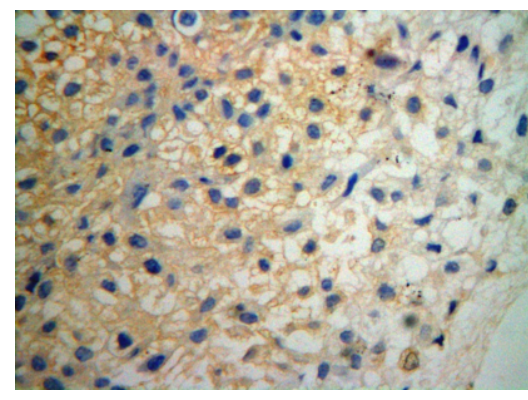

Fig. 4. $\mathrm{Cx} 43$ protein expression in the human placental decidual cells at 7 weeks of pregnancy, $\mathrm{SABC} \times 400$.

\subsection{Pax3 expression in the human placental villi and decidua during early pregnancy}

Pax 3 was mainly distributed in the cytoplasm, nucleus and cell membrane of placental trophoblast cells and decidual cells. From 5 to7 weeks of pregnancy, the IOD of Pax3 protein expressed in the syncytiotrophoblast decreased gradually (Figures 5 and 6 ), and the differences between 5 and 6 weeks were statistically significant $(\mathrm{P}<0.05)$. However, Pax3 in cytotrophoblast cells and decidual cells showed increased IOD from 5 to 7 weeks (Figure 7 and 8), and the differences were statistically significant $(\mathrm{P}<0.05) \quad$ (Table 2$)$.

Table 2

Pax3 expression in the human placental villi and decidua during early pregnancy

\begin{tabular}{lllll}
\hline \multirow{2}{*}{$\begin{array}{l}\text { Group } \\
\text { cases }\end{array}$} & Length of pregnancy & \multicolumn{4}{l}{ The average of positive Pax3 IOD (mean \pm SD) } \\
\cline { 2 - 5 } & & Syncytiotrophoblast & Cytotrophoblast & Decidual cells \\
\hline 8 & 5 weeks & $2492.89 \pm 302.59$ & $2397.78 \pm 278.06$ & $2355.38 \pm 295.38$ \\
\hline 10 & 6 weeks & $2198.44 \pm 325.87$ & $2524.46 \pm 289.05$ & $2473.55 \pm 297.40$ \\
\hline 8 & 7 weeks & -- & $2873.47 \pm 292.11$ & $2944.63 \pm 443.39$ \\
\hline F 值 & & 3.861 & 5.969 & 6.531 \\
\hline P & & 0.067 & 0.008 & 0.006 \\
\hline
\end{tabular}

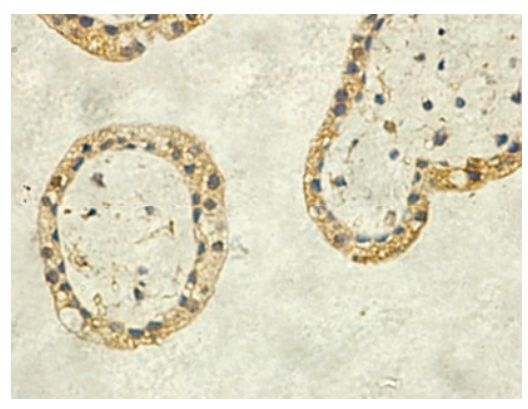

Fig. 5. Pax3 protein expression in the human placental trophoblast at 5 weeks of pregnancy, SABC $\times 400$.

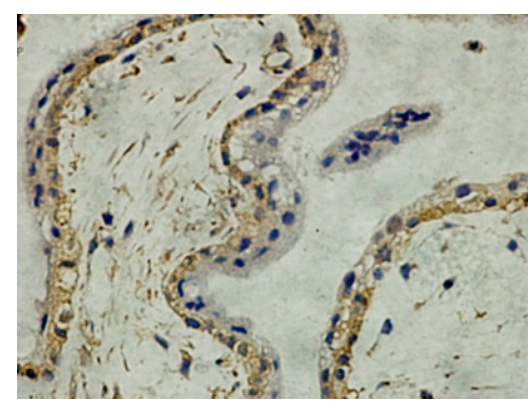

Fig. 6. Pax3 protein expression in the human placental trophoblast at 7 weeks of pregnancy, $\mathrm{SABC} \times 400$. 


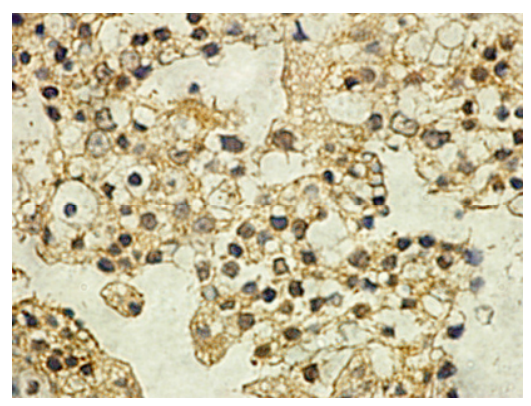

Fig. 7. Pax 3 protein expression in the human decidual cells at 5 weeks of pregnancy, $\mathrm{SABC} \times 400$.

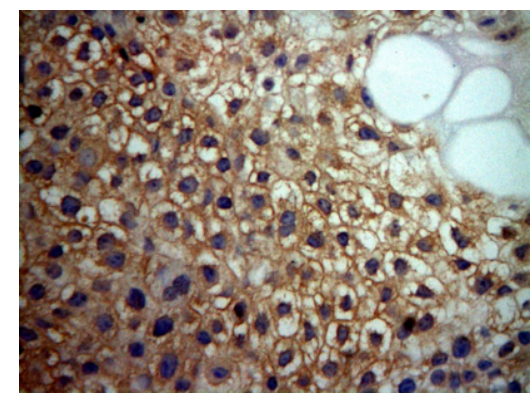

Fig. 8. Pax3 protein expression in the human decidual cells at 7 weeks of pregnancy, $\mathrm{SABC} \times 400$.

\section{Discussion}

The normal development of the placenta plays an important role in the maintenance and progress of pregnancy. One major step for placenta formation is the fusion of cytotrophoblast with syncytiotrophoblast. The syncytiotrophoblast layer on the villous surface directly invades the uterine wall, rupturing maternal capillaries and thus participating in material exchange between the mother and the fetus. Moreover, the syncytiotrophoblast secretes steroid and peptide hormones. Hence, it plays a major role in the above mentioned function, and is essential for fetal development. $\mathrm{Cx} 43$ expression determines the formation of gap junctional intercellular communications (CJIC) and decides the structure and number of communication channels. It also affects the fate of channels. Early embryos without the completely established nervous and circulatory systems are not capable for longdistance information transmission and regulation. The intercellular exchange of chemical signals is required for the proliferation, differentiation and development of diverse embryonic cells. Therefore, $\mathrm{Cx} 43$ is a structural protein controlling intercellular gap junctions. Its expression is closely related to multiple biological processes such as embryonic development, cell induction, differentiation, growth regulation and apoptosis [2-4]. Pax belongs to an evolutionarily conserved gene family and widely exists in various organisms. Pax gene encodes a protein distributed in the nucleus, which is an important transcription factor. It plays a critical regulation role in the specification of tissues and organs during embryonic development $[5,6]$. Rong Xiao et al. revealed that cyclophosphamide inhibits Pax 3 protein expression and meanwhile promotes $\mathrm{Cx} 43$ overexpression. They proposed that Pax 3 is likely to be the negative regulatory factor for $\mathrm{Cx} 43$ [7]. However, our results are not consistent with the above idea. Our data showed that at 5 weeks of pregnancy, Cx43 and Pax3 proteins were positively expressed in the human syncytiotrophoblast, cytotrophoblast and villi central axis cells. From 5 to 7 weeks, the IOD values of $\mathrm{Cx} 43$ and Pax3 proteins gradually increased in the cytotrophoblast, while the IOD values in the syncytiotrophoblast showed a decreased trend. After 7 weeks, the syncytiotrophoblast did not express $\mathrm{Cx} 43$ and Pax3 proteins. We propose that the villous trophoblast is divided into the cytotrophoblast and syncytiotrophoblast during early pregnancy. The villi central axis cells are differentiated into various types of cells such as connective tissue cells and vascular endothelial cells. There is no nervous system to regulate the differentiation and function of different placental cells. GJIC is necessary for regulating the nutrients and control signals required by cell proliferation, differentiation, growth and morphology. Along with the villous development and placental formation, $\mathrm{Cx} 43$ and Pax3 expression is enhanced in the cytotrophoblast. Syncytiotrophoblast cells cannot proliferate, so dead or apoptotic syncytiotrophoblast cells are 
replaced by cytotrophoblast cells, which have the proliferation ability. The syncytiotrophoblast forms as a result of differentiation of the cytotrophoblast cells. It suggests that $\mathrm{Cx} 43$ and Pax3 proteins play an important role in the differentiation and development of the cytotrophoblast. The expression of $\mathrm{Cx} 43$ and Pax3 and their relationship with pregnancy weeks are synchronous with the differentiation of the cytotrophoblast. Hence, we think that the expression levels of $\mathrm{Cx} 43$ and Pax3 are associated with the differentiation of the cytotrophoblast.

During human reproduction, the blastocyst obtains enough nutrition in the uterus and develops into an embryo. The trophobast is the outer layer of the blastocyst. It plays an important role in invading or breaking the endometrial epithelial barrier and consequently contacting with endometrial stroma and maternal blood [8]. Decidual cells are rapidly differentiating, glycogen-enriched cells from the mucous membrane. The process of decidual transformation is required for successful pregnancy [9]. In the present study, we showed that $\mathrm{Cx} 43$ and Pax3 proteins were expressed in decidual cells. From 5 to 7 weeks of pregnancy, the IOD values of $\mathrm{Cx} 43$ and Pax3 proteins expressed in decidual cells gradually increased and reached the peak at 7 weeks. It indicates that $\mathrm{Cx} 43$ and Pax 3 have increased impact on the regulation of decidua during the decidual process. It implicates that $\mathrm{Cx} 43$ and Pax 3 expression and their relationship with pregnancy weeks are synchronous with the decidual differentiation. Decidual cells exchange materials and information through GJIC. Hence, we propose that the expression levels of $\mathrm{Cx} 43$ and Pax3 are associated with the decidual process and may be important for placental formation, development and function.

\section{Conclusion}

In summary, we suggest that the expression levels of $\mathrm{Cx} 43$ and Pax3 are related to cell differentiation and structural formation of placental villi as well as the decidual differentiation. The synchronous expression of $\mathrm{Cx} 43$ and Pax3 with the human placental villi and decidua indicates that Cx43 and Pax3 may play a synergistic role and participate in the regulation of endometrial decidualization and trophoblast differentiation and invasion. They may be essential for cell proliferation and differentiation during the processes of placental formation and the generation and development of placental villi.

\section{References}

[1] Dongwei Mao, Dongmei Zheng, Fang Li, Yixuan Zhao and Jianhua Che, The effect of taurine on diabetes during pregnancy and the expression of development-related genes in the placenta, Shenyang Budui Yiyao 18 (2005), 251-253.

[2] D.V. Krysko, S. Mussche, L. Leybaert and K. D'Herde, Gap junctional communication and connexin43 expression in relation to apoptotic cell, death and survival of granulose cells, J. Histochem. Cytochem. 52 (2004), 1199-1207.

[3] A. Malassine and L. Cronier, Involvement of gap junctions in placental functions and development, Biochim. Biophys. Acta. 1719 (2005), 117-124.

[4] M.S. Holland, J.A. Stasko and R.E. Holland, Influence of extracellular matrix on bovine mammary gland progenitor cell growth and differentiation, Am. J. Vet. Res. 68 (2007), 476-482.

[5] M. Buckinqham and F. Relaix, The role of Pax genes in the development of tissues and organs: Pax3 and Pax7 regulate muscle progenitor cell functions, Annu. Rev. Cell Dev. Biol. 23 (2007), 645-673.

[6] Dongwei Mao, Yingjie Zhang, Qiumei Li and Shourou Li, Expression of Pax3 and Cx43 in NTD embryos induced by hyperglycemia, National Medical Journal of China 83 (2003), 593-597.

[7] Rong Xiao, Haifeng Zhao, Xiuhua Li, Xuemin Li, Zhiying Cui and Geng Gao, Effect of soybean is of lavone combining with folate on the expression of Pax3 and $\mathrm{Cx} 43$ in neurons of rat embryo with neural tube defects, Acta Nutriment Sinica 29 (2007), 178-180. 
[8] L. Marx, P. Arck, M. Kapp, C. Kieslich and L.J. Dietl, Leukocyte populations, hormone receptors and apoptosis in eutopic and ectopic first trimester human pregnancies, Hum. Reprod. 14 (1999), 1111-1117.

[9] Gang Wang and Zhen Han, Growth regulation of decidua during early pregnancy, Chinese Journal of Woman and Child Health Research 10 (1999), 75-78. 